\title{
In Ovo Progression of Borderline Ovarian Tumors
}

\section{Meynier $\mathbf{S}^{1}$, Undurraga $\mathbf{M}^{1}$, Tille $\mathrm{JC}^{2}$, Petignat $\mathrm{P}^{1}$ and Cohen $\mathbf{M}^{1 *}$}

${ }^{1}$ Department of Gynecology Obstetrics, Faculty of Medicine, University of Geneva, 30 bd de la Cluse, 1211 Geneva 14, Switzerland 2Division of Clinical Pathology, Geneva University Hospital, Rue Michel-Servet 1, 1211 Geneva 4, Switzerland

\begin{abstract}
Objective: Borderline ovarian tumors (BOTs) are generally considered to be non-invasive tumors with low malignancy potential. However, studies show that BOTs have the ability to progress to low-grade carcinomas and gain the potential to invade surrounding tissue and to metastasize. Here, we investigate whether a patient derived serous BOT (SBOT) could progress in low-grade serous carcinoma (LGSC) through in vitro and in ovo characterization.
\end{abstract}

Methods: Ovarian cancer cell line from a SBOT, named OCAM, were isolated and characterized by PCR, migration assay and chick chorioallantoic membrane (CAM) assay.

Results: OCAM cells express carcinoma characteristics and form a tumor in CAM. Moreover, OCAM cells get into membrane of chicken suggesting invasive properties.

Conclusion: SBOTs could a precursor of LGSC involving consequences in adaptation of treatment provided by clinician.

Keywords: Borderline tumor; Ovarian; Low grade; Progression

\section{Introduction}

Borderline ovarian tumors (BOTs), also named atypical proliferative ovarian tumours (APOTs), were first recognized in 2003 [1]. BOTs are viewed as an intermediate state between benign cystadenomas and adenocarcinomas. BOTs represent $15-20 \%$ of epithelial ovarian carcinomas (EOC), and $80 \%$ of them are diagnosed during the early stages of development [2,3]. Most women are diagnosed around 40 years of age but this can vary depending on ethnicity and geography [4]. Histological characteristics of BOTs are well-formed papillae with epithelial stratification and mild to moderate cellular atypia [5-7].

Patients diagnosed with stage I BOTs have excellent 5-year survival rates of between $90 \%$ and $100 \%$, but this rate decreases when discovered in advanced stages [4]. In most cases, BOTs are asymptomatic and diagnosed during routine examination. The standard treatment for BOTs in advanced stages requires surgically removing the macroscopically visible tumor. Adjuvant therapy for advanced stages treatment remains controversial [8]. Risk factors such as age, histology, and type of surgery (for example fertility sparing surgery); appear to be associated with a higher risk for recurrence. Histologic parameters such as peritoneal implants, microinvasion, and micropapillary pattern do not seem to be associated with a higher recurrence rate $[9,10]$. Tumor markers, such as CA-125 and CA 19-9 are increased in BOTs, with a higher increase of CA-125 in serous BOT (SBOT) and CA 19-9 in MBOT [11].

Recent studies show that SBOTs have the potential to become a low-grade serous carcinoma (LGSC) and are associated with molecular modifications or microsatellite instability [12-14]. SBOTs and LGSCs share common mutations in KRAF, BRAF, ERBB2 supporting the hypothesis that SBOT might potentially progress to LGSC [14-16]. The hypothesis suggesting that BOTs are a precursor state for LGSC is now accepted, although clinical and molecular studies are still necessary to understand the mechanism and risk factors.

In this report, we isolated a clonal cell line established from a SBOT, termed OCAM, belonging to a 21 year old patient. OCAM cells were characterized and its ability to migrate was evaluated. Moreover, the capacity of OCAM to grow and to invade was investigated using chick chorioallantoic membrane (CAM) assay.

\section{Materials and Methods}

\section{Patient and clinical background}

A 21 year old asymptomatic white female consulted a gynecologist for contraception prescription. She has no particular medical history. She smoked 10 packs per year. Her physical examination was significant for a distended abdomen, with a palpable abdominal mass. Abdominal and pelvic computed tomographic (CT) scans and MRI confirmed the clinical evaluation of abnormal pelvic mass. Bilateral ovarian masses infiltrating the left paracolic gutter as well as a moderate quantity of ascites were noticed. Tumor markers were as follows: CA 125: $516 \mathrm{kU} / \mathrm{l}$, Alpha-feto-protein: 3.7 uj/l, LDH 146 U/l.

An exploratory laparoscopy converted into laparotomy followed by fertility-sparing surgery was performed. It included a right salpingo-oophorectomy, left ovarian cystectomy, omentectomy and appendectomy. The patient post-operative period was uneventful and she was discharged one-week post-op. Final pathological analysis showed serous borderline ovarian tumor with micro-invasion focus, FIGO stage of IIIB. Close follow-up was performed and 18 months later, a right ovarian mass with normal CA 125 was identified. The patient opted for left salpingo-oophorectomy, and pathology confirmed recurrence of a serous borderline ovarian tumour (SBOT) (Figure 1). Three years after the second surgery, no sign was observed.

*Corresponding author: Cohen M, Department of Gynecology and Obstetrics, Faculty of Medicine, University of Geneva, 30 Boulevard de la Cluse, 1205 Geneva, Switzerland, Tel: +41 223824 381; Fax: +41 223824 310; E-mail: marie.cohen@hcuge.ch

Received August 15, 2016; Accepted September 06, 2016; Published September 13,2016

Citation: Meynier S, Undurraga M, Tille JC, Petignat P, Cohen M (2016) In Ovo Progression of Borderline Ovarian Tumors. J Oncol Transl Res 2: 109.

Copyright: $\odot 2016$ Meynier S, et al. This is an open-access article distributed under the terms of the Creative Commons Attribution License, which permits unrestricted use, distribution, and reproduction in any medium, provided the original author and source are credited. 

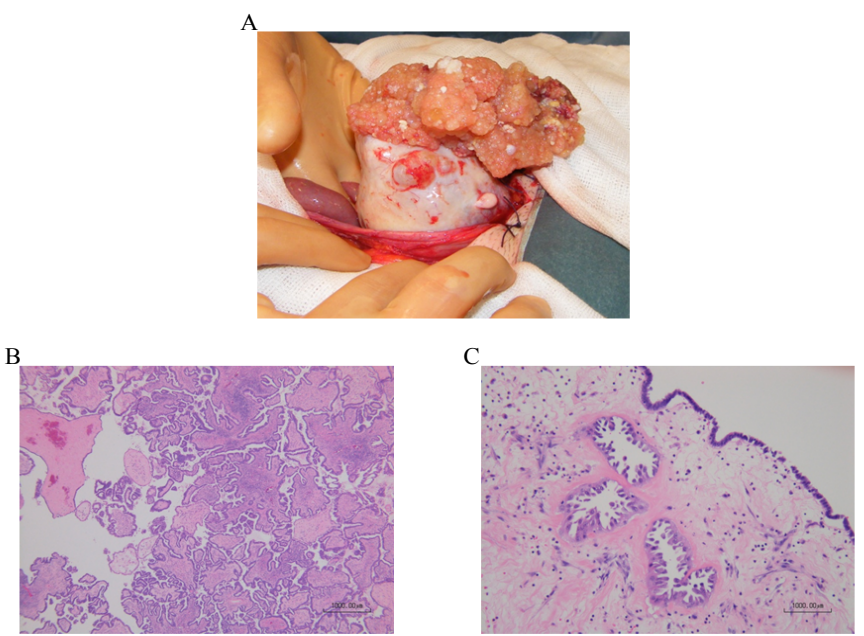

Figure 1: A- Operative picture of tumor; B: HE staining of the borderline region (magnification $\mathrm{x} 40$ ), C- HE staining of the micro-invasive region (magnification $\mathrm{x} 200)$.

\section{Establishment of the ovarian cancer cell line: OCAM}

Ovarian tissue was digested with $4 \mathrm{mg} / \mathrm{ml}$ dispase (Gibco, Invitrogen, Basel, Switzerland) in HBSS-HEPES (filtered on $22 \mu \mathrm{m}$ ) containing $1 \mu \mathrm{g} / \mathrm{ml}$ DNase (Roche, Diagnostics GmbH, USA) for 30 min at $37^{\circ} \mathrm{C}$. Ovarian tissue and supernatant were placed into $10 \mathrm{~cm}$ dishes and the tissue was scrubbed with a scalpel. The supernatant was collected, neutralized with 5\% FBS, filtered through a $100 \mu \mathrm{m}$ mesh (BD Biosciences, San Jose, USA) and centrifuged at $800 \mathrm{~g}$ for $8 \mathrm{~min}$. The resulting pellet was resuspended in DMEM containing 10\% FBS and $0.05 \mathrm{mg} / \mathrm{ml}$ gentamicin and cells were seeded in a $3 \mathrm{~cm}$ dish. The medium was replaced every two days. Cells were detached by using $0.25 \%$ trypsin-EDTA and were transferred into a $10 \mathrm{~cm}$ dish. OCAM cells were obtained by limiting dilution in a 96-well plate at passage 2 . The cells were sub cultured every 5 days at a dilution 1:8.

\section{Cell culture}

OCAM cell line was cultured at $37^{\circ} \mathrm{C}$ under $5 \% \mathrm{CO}_{2}$ in Roswell Park Memorial Institute medium 1640 (RPMI 1640, Gibco, Invitrogen,
Basel, Switzerland) containing 10\% fetal bovine serum and $0.05 \mathrm{mg} /$ ml gentamicin.

\section{Reverse transcription polymerase chain reaction (RT-PCR)}

Expression of CD90, PAX8, KILK6, KRT7, KRT8, KRT19, Cytovillin, ESR1, ESR2, CLEC11A, HTRA1, COL12A1, COLT1, SLPI and HE4 mRNA were investigated in OCAM cells. Reverse transcription was performed with $1 \mu \mathrm{g}$ of total RNA in a final volume of $20 \mu \mathrm{l}$ using High Capacity cDNA Reverse Transcription Kit (Applied Biosystems, Foster City, USA). The detection of the PCR product was performed using REDTaq "ReadyMix " PCR Reaction Mix from Sigma Aldrich (St Louis, MO, USA), with the Thermal cycler from Bioer (BioConcept, Switzerland). The experiment was performed in duplicate.

Oligonucleotide primers for PCR are listed in Table 1.

\section{Wound healing assay}

$1 \times 10^{5}$ OCAM cells were seeded in a 12 -well plate. Twelve hours after, cells were treated with $2.5 \mu \mathrm{g} / \mathrm{ml}$ of mitomycin for $2 \mathrm{~h}$. Then, mitomycin solution was removed, a straight scratch was performed with pipette tip stimulating a wound and medium was added. At different time points $(0,2,4,6,8,10,24 \mathrm{~h})$, a picture was assessed using xlicap software. The experiment was performed in triplicate.

\section{Immunocytochemistry}

OCAM cells were seeded into Lab-tek chamber slide $\left(2 \times 10^{4}\right.$ cells /well) and cultured in RPMI medium. After $24 \mathrm{~h}$, cells were washed in Phosphate Buffer Saline (PBS), fixed in $4 \%$ Paraformaldehyde (PFA) for $15 \mathrm{~min}$ and washed 3 times in PBS. Cells were permeabilised with $0.2 \%$ Triton X-100 in PBS for 10 min. Afterwards, non-specific sites were blocked with RPMI medium for $30 \mathrm{~min}$ and then incubated overnight at $4^{\circ} \mathrm{C}$ with mouse monoclonal antibodies against cytokeratin 18 (dilution 1:50, clone DC10, Dako, Baar, Switzerland), mouse monoclonal antibodies against cytokeratin 7 (dilution 1:50, clone OV-TL 12/30, Dako, Baar, Switzerland), mouse monoclonal antibodies against cytokeratin 19 (dilution 1:50, BA17, Santa Cruz Biotechnology, San Diego, CA, USA), mouse monoclonal antibodies against p21 (dilution 1:50, clone SX118, Dako, Baar, Switzerland), mouse monoclonal antibodies against p53 (dilution 1:50, Ab-3, Calbiochem, Merk Millipore, Switzerland) or mouse IgG (dilution 1:50, sc-2025, Santa Cruz Biotechnology, San Diego, CA, USA). Cells were

\begin{tabular}{|c|c|c|}
\hline & Forward (5'-3') & Reverse (5'-3') \\
\hline CD90 & ATCGCTCTCCTGCTAACAGT & ACTTGACCAGTTTGTCTCTGA \\
\hline CLEC11A & ACCTCTTCGAAAACGGCCAG & GCAGACGTAGTAGAGACGCC \\
\hline COL12A1 & CCTACAACGGATGGGCCTAC & ACTGAGCGTGGTTGTCTGAG \\
\hline COLT1 & TCCCTACTAAGGCCCAGACC & ССTTCTCACCACCGAGCAAT \\
\hline Cytovillin & AGGCGGTGGATCAGATAAAGA & СTCCTGGACATGGTAGCTCAC \\
\hline ESR1 & CCTTTGGCCAAGCCCGCTCA & TGGAGCGCCAGACGAGACCA \\
\hline ESR2 & GGTCGGCAGACCACAAGCCC & TAACAGGGCTGGCGCAACGG \\
\hline HE4 & CGGCTTCACCCTAGTCTCAG & ССТССTTATCATTGGGCAGA \\
\hline HTRA1 & CCAGACGTGATCTCAGGAGC & ACGGGAGAGTCTTTGAGGGA \\
\hline KLK6 & AGCCAAACTCTCTGAACTCAT & AGTGATGCAAGGATGGAGCT \\
\hline KRT19 & ACTACAGCCACTACTACACGACCAT & GTCTCAAACTTGGTTCGGAAGTC \\
\hline KRT7 & TTGCCTCCTTCATCGACAAGGTGC & TCATACTGCGCCTTGACCTCAGCG \\
\hline KRT8 & AACAACCTTAGGCGGCAGCT & GCCTGAGGAAGTTGATCTCG \\
\hline PAX8 & АССTTCTCCATGTGGGACAC & GGATGCTGTGCTCAAAGTGA \\
\hline SLPI & CCTGGATCCTGTTGACACCC & ACAGGGGAAACGCAGGATTT \\
\hline CYCLOPHILIN A & ATGGTCAACCCCACCGTG & TGCAATCCAGCTAGGCATG \\
\hline
\end{tabular}


then washed 3 times in PBS and incubated for 1 hour with goat antimouse IgG-HRP (dilution 1:500 Santa Cruz Biotechnology, San Diego, CA, USA). After washing, cells were stained with diaminobenzidine (DAB) chromogen system (Dako, Baar, Switzerland) following by a hemalun counterstaining.

To perform this, Mayer's Hemalun solution (Merk Millipore, Switzerland) was diluted to $1 / 4$ in water. The solution was then applied to the sample for $1 \mathrm{~min}$ and immerged in warm water. The slide was then dried, Aquatex (Merk Millipore, Switzerland) was added and a glass coverslip was put onto the slide.

\section{Tumor development on chick chorioallantoic membrane (CAM)}

On Embryo Development Day 1 (EDD1), fertilized eggs (animal facility of the University of Geneva, Geneva, Switzerland) were incubated at $38^{\circ} \mathrm{C}$ with $80 \%$ relative humidity and periodic rotation.

On EDD4, rotation was stopped and eggs were drilled at their narrow apex. The hole was closed with adhesive tape. Incubation was carried out until use.

On EDD8, after gentle scratching of the membrane with a needle tip, a silicon O-ring (Apple Rubber products Inc., Lancaster, USA) was placed onto a blood vessel crossing. OCAM cells were resuspended at $2.5 \times 10^{6}$ cells in $30 \mu \mathrm{l}$ of RPMI- 1640 (containing $10 \%$ fetal bovine serum and $0.05 \mathrm{mg} / \mathrm{ml}$ gentamicin). The suspension was then placed into the silicon O-ring. The hole was hermetically covered with Parafilm and eggs were returned to the incubator.

On EDD12, tumor growth was monitored using a Wild Heerbrugg M3Z microscope at 10x magnification with a Lumenera INFINITY2-1 CDD camera with Infinity Capture Software.

The ovarian tumor was rapidly washed with $0.1 \mathrm{M}$ phosphate buffered saline (PBS) at $\mathrm{pH} 7.4$ and fixed in $4 \%$ buffered formalin at $4^{\circ} \mathrm{C}$ for $24 \mathrm{~h}$. The specimens were then dehydrated in ethanol and embedded in paraffin wax.

\section{Hematoxylin and eosin staining (HE)}

The section of tissue was deparaffinised and rehydrated through graded ethanol before being immersed in the filtered Harris Hematoxilin solution modified (Sigma Aldrich, St Louis, MO, USA) for $1 \mathrm{~min}$ and rinsed with tap water. The section was immersed in eosin for $1 \mathrm{~min}$, rinsed with tap water and was then dehydrated with a series of ethanol solutions: $50 \%, 70 \%, 80 \%$, two baths with $95 \%$ and two baths with $100 \%$. To finish, one bath of xylene for $5 \mathrm{~min}$ was performed. Then, Aquatex was added and a glass coverslip was put on slide.

\section{Immunohistochemistry}

Serial sections of tissue were deparaffinised and rehydrated through graded ethanol as described previously. Antigen retrieval was performed by microwave pre-treatment in $10 \mathrm{mmol} / \mathrm{l}$ citrate buffer $(\mathrm{pH}$ 6.0) for 5 min four times, followed by cooling in a cold water bath. Non-specific binding was blocked with $3 \%(\mathrm{v} / \mathrm{v})$ bovine serum albumin (BSA) in PBS for $30 \mathrm{~min}$ at room temperature. The sections were incubated with mouse monoclonal antibodies against p21 (dilution 1:50), mouse monoclonal antibodies against p53 (dilution 1:50), mouse monoclonal antibodies against Ki-67 (dilution 1:50, BD Sciences, Allschwill, Switzerland) or mouse IgG (dilution 1:50). Sections were then washed with PBS and incubated with goat anti-mouse IgG-HRP (dilution 1/500 in 3\% BSA-PBS) for $1 \mathrm{~h}$. After washing, sections were stained with diaminobenzidine (DAB) chromogen system following by a hemalun counterstaining. Aquatex (Merk Millipore, Switzerland) was added and a glass coverslip was put on slide.

\section{Results}

\section{OCAM characterization through 15 genes}

To characterize OCAM cell line, we investigated the expression of different genes known to participate in cancer cell invasion (keratin, KLK6, SLPI), growth (PAX8), adhesion (cytovillin, CD90), proliferation (HE4, oestrogen receptor, CLEC11A, COLT1, COL12A1) and chemoresistance (HTRA1). Most of these genes are currently studied for the characterization of ovarian cancer cell line, with the exception of CLEC11, HTRA1, COL12A1, COLT1 and SLPI, which were previously identified in our lab to be differentially expressed in borderline ovarian cancer cells compared to serous ovarian cancer cell.

OCAM cell line was positive for the following genes: PAX8, KRT8, KRT19, Cytovillin, KRT7, CLEC11A, HTRA1, COL12A1, SLPI and HE4 (Table 2). On the other hand, OCAM cells were negative for CD90, KLK6, ESR1, ESR2 and COLT1 genes.

\section{Tumour markers}

Immunocytochemistry was performed to assess the expression levels of known oncoproteins p21, p53 as well as proliferative markers cytokeratin 7, cytokeratin 18 and cytokeratin 19 in OCAM cells. As observed in, OCAM cells were negative for p21. OCAM cells were also negative for CK19 while they expressed KRT19 mRNA. We also observed a heterogenous nuclear staining for p53 with a variable intensity an intense cytoplasmic staining for CK7 and a slight cytoplasmic staining for CK18 (Figure 2).

\section{OCAM migration}

To examine the ability of OCAM cells to migrate, a wound-healing assay was performed. OCAM cells covered the half of the wound within $24 \mathrm{~h}$ thus showing their ability to migrate with $50 \%$ closure after $24 \mathrm{~h}$ (Figure 3).

\section{OCAM tumor development}

To further investigate the ability of OCAM cells to migrate and to invade into surrounding tissues in ovo, OCAM cells were inoculated on CAM. Seven days after the inoculation, the formation of a tumor was

\begin{tabular}{|c|c|}
\hline & OCAM \\
\hline CD90 & - \\
\hline CLEC11A & + \\
\hline COL12A1 & + \\
\hline COLT1 & - \\
\hline Cytovillin & + \\
\hline ESR1 & - \\
\hline ESR2 & - \\
\hline HE4 & + \\
\hline HTRA1 & + \\
\hline KLK6 & - \\
\hline KRT19 & + \\
\hline KRT7 & + \\
\hline KRT8 & + \\
\hline PAX8 & + \\
\hline SLPI & + \\
\hline
\end{tabular}

+: expressed, -: not expressed

Table 2: Characterization of OCAM cell line mRNA by RT-PCR. 
A
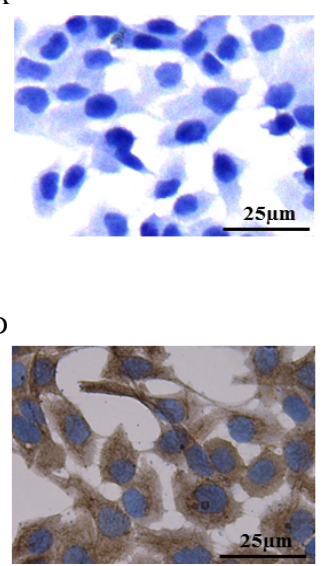

B

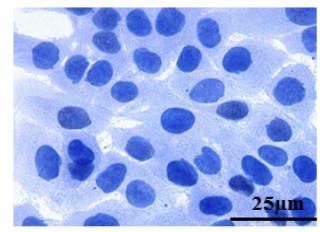

E

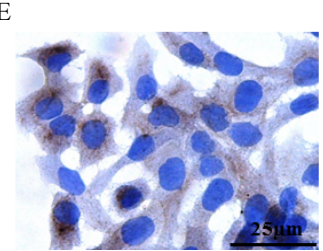

$\mathrm{C}$

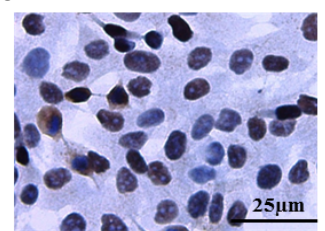

F

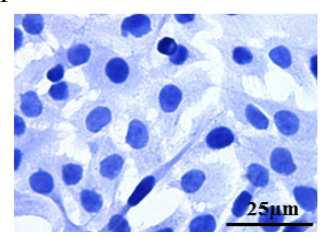

Figure 2: Tumor markers immunostaining of OCAM cells for mouse IgG as control (A), p21 (B), p53 (C), CK7 (D), CK18 (E) and CK19 (F). The magnification used is $\times 400$.

A

T0

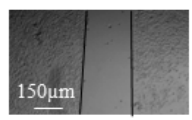

T2

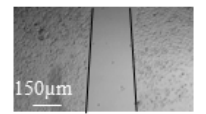

T4

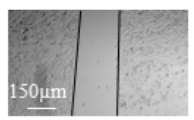

T6

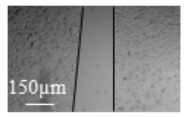

T8

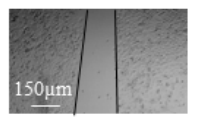

$\mathrm{T} 10$

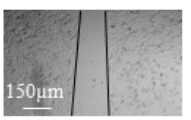

T24

B

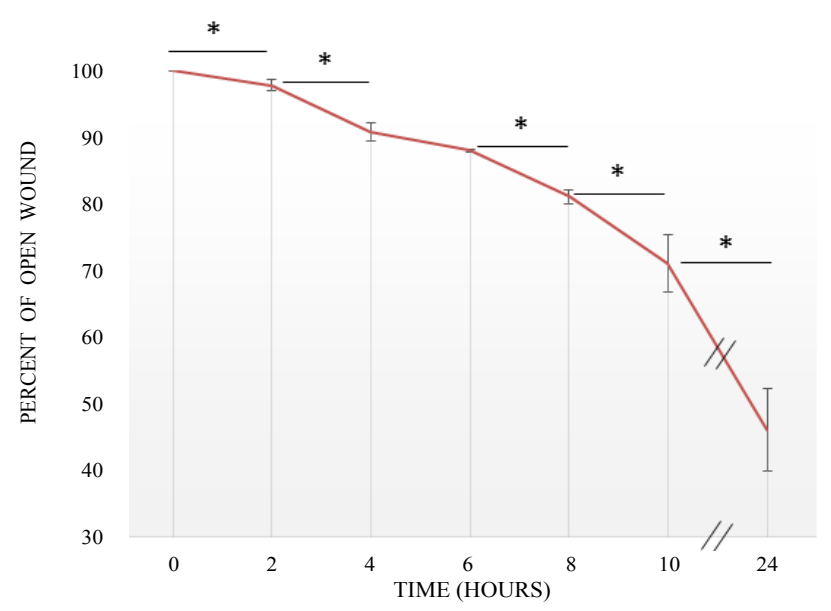

Figure 3: OCAM migration capacity OCAM cells were seeded in 12-well plate. The next day, cells were treated with mitomycin for $2 \mathrm{~h}$ to block cell proliferation and then a straight scratch was performed. To evaluate cell migration, a picture was assessed at different incubation time (0, 2, 4, 6, 8, 10, $24 \mathrm{~h})(\mathrm{A})$. Figure B represents the quantification of open wound after the scratching $\left({ }^{*} p<0.05\right.$, Student t-test).

observed with several vessels converging to the tumor, thus showing the presence of a vascular network able to supply blood to the tumor.

At EDD 12, the tumor was removed embedded in paraffin and HE staining was performed. OCAM cells have formed a tumor (designated by number 2) next to the membrane of CAM (designated by number 1). The tumor is formed of small papillae with epithelial stratification and small regular nucleus, which is the characteristic of a low-grade tumor. Moreover, these cells seem to have infiltrated the membrane of the chicken. Immunohistochemistry further showed negative staining for p21 and very low Ki67, proliferation marker and a heterogeneous positive staining for wild type p53 (Figure 4).

\section{Discussion}

Borderline ovarian tumors were long considered to be of benign evolution. It was proved this is not true, with overall survival (OS) rates lower than the general population in patients with advanced stage disease [17]. It is not clear if this decrease in OS is due to the borderline tumor per se, or to those tumors, that with time, mutate to low grade ovarian tumors, which are known to have very low response rates to systemic therapies. Unfortunately, to this day there is no way of identifying which patients will recur and if the recurrence will be as borderline tumor or low grade ovarian tumors.

This study report a cell line evaluation from a patient with a 
A

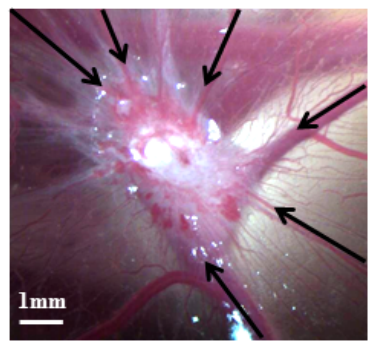

ii
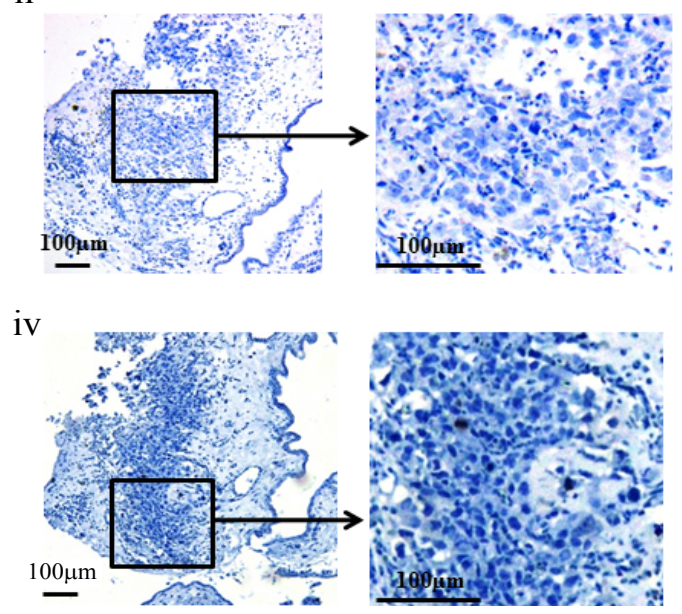

$\mathrm{B}$

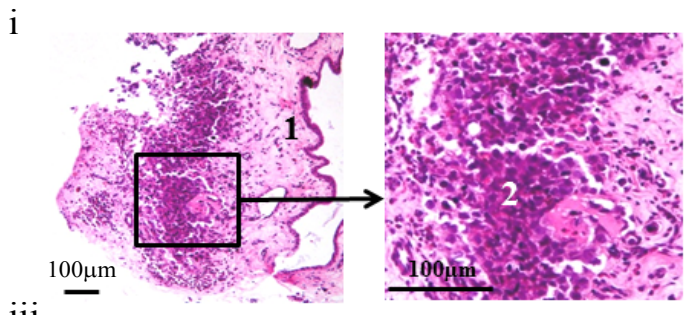

iii
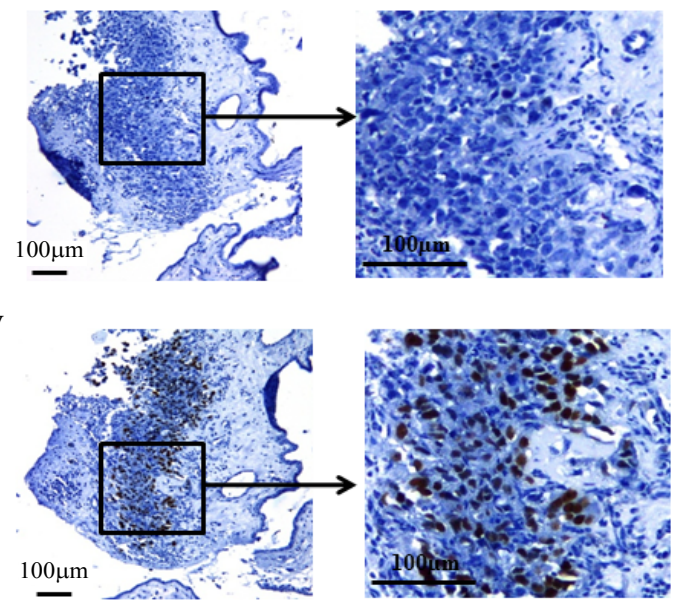

Figure 4: OCAM tumour development A-On EDD8, after gently scratching of CAM, OCAM cells suspension was placed into the silicon O-ring. On EDD12, OCAM tumor was observed and a picture of tumor was taken. Arrows indicate the vascular network around the tumor. Bi- $\mathrm{HE}$ staining of OCAM tumor: 1 . membrane of CAM; 2. OCAM tumor. B- Immunostaining of OCAM tumor for mouse IgG (ii), p21 (iii), Ki67 (iv) and p53 (v). The magnification used is x200. Each square represents an enlargement of the same location.

SBT named OCAM, and investigated its characteristics in vitro and in ovo. From a genetic point of view, we found that OCAM cell line is positive for PAX8, KRT8, KRT19, Cytovillin, KRT7, CLEC11A, HTRA1, COL12A1, SLPI and HE4 genes. These genes are known to participate in cell invasion, growth, cell adhesion, proliferation and chemoresistance, demonstrating the tumoral potential of this cell line. Moreover, we also observed that OCAM cells are highly proliferative in vitro (data not show). 553 expression was then investigated in these cells by immunocytochemistry. p53 is a well-known tumor suppressor which is inactivated in most cancer cells. Most of BOTs and LGSC were very rarely positive for $\mathrm{p} 53$ mutations and were almost wild type for $\mathrm{p} 53$ staining [18-21]. In contrast, most high grade serous ovarian cancers present a high expression of mutated p53 and a strong expression of p53 staining [18]. In our study, a heterogeneous nuclear expression of p53 was observed in OCAM cells which discriminates the high grade serous ovarian cancer. However, the heterogeneous character of p53 staining in vitro and in ovo is characteristic of BOTs and LGC. At the moment, the difference between SBOTs and LGSC is based on the invasive character of the tumor [7]. In ovo, OCAM cells have the capacity to grow and to form a tumor on the membrane and to get into the membrane of chicken showing thus a potential of invasion (Figure 4). These different characteristics could suggest the potential ability of SBOT to evolve in LGSC.

Risk factors associated with SBOTs progressing to LGSC may have important implications for the clinician, who may have to evaluate the potential benefit of adjuvant therapy, follow-up and treatment of recurrences. At the moment, most investigators do not prescribe adjuvant therapy for patients with BOTs due to its low response rate.
However, the presence of vessel proliferation around OCAM tumor may potentially change the practice as the use of new anti-angiogenesis target-based therapies as adjuvant settings. Sprouting angiogenesis is the most important mechanism for tumour vascularisation and thus tumour proliferation. The presence of a specific pathway that may be targeted, such as VEGF or its receptors, could open the way for directed therapies. The ability for angiogenesis in this line of BOT would explain why some patients with BOTs and LGSC treated with anti-angiogenic therapy (bevacizumab), with or without chemotherapy, have a survival improvement as compared to chemotherapy alone [22]. Another important issue is the confirmation of the migration of these cells. This capacity puts patients at risk who are considered R0 after surgery, and may have an impact not only in adjuvant therapy, as discussed above, but also in follow-up. To date, no definite follow-up strategies have been established, CA-125 and loco-regional ultrasound being the most used, but this capacity of migration by the tumoral cells may open the need for wider imaging [23].

\section{Conclusion}

Molecular studies have already shed light on the relationship between BOTs and low grade ovarian tumors. Further molecular and cellular studies on human tissue are now needed to establish which patients will evolve from BOTs to low grade tumors, thus better orienting treatment and follow-up.

\section{Acknowledgement}

We wish to thank Ginette Rosseel (Department of Gynecology and Obstetrics, Geneva University of Hospital) for the recruitment of patients, Kylie Van Hoesen, Marianne Kramer and Dr. Robert Bradshaw for the reviewing and the Fonds 
National Suisse de la Recherche Scientifique (FNRS, 31003A-127392) for financial support.

\section{References}

1. Tavassoli FA, Peter D (2003) World Health Organization Classification of Tumours. Pathology and Genetics Tumours of the Breast and Female Genital Organs. IARC Press: Lyon.

2. Lenhard MS, Mitterer S, Kümper C, Stieber P, Mayr D, et al. (2009) Long-term follow-up after ovarian borderline tumor: Relapse and survival in a large patient cohort. Eur J Obstet Gynecol Reprod Biol 145: 189-194.

3. Trillsch F, Mahner S, Ruetzel J, Harter P, Ewald-Riegler N, et al. (2010) Clinical management of borderline ovarian tumors. Expert Rev Anticancer Ther 10: 1115-1124.

4. Lalwani N, Shanbhogue AK, Vikram R, Nagar A, Jagirdar J, et al. (2010) Current update on borderline ovarian neoplasms. AJR Am J Roentgenol 194: 330-336.

5. Patrono MG, Minig L, Diaz-Padilla I, Romero N, Moreno JFR, et al. (2013) Borderline tumours of the ovary, current controversies regarding their diagnosis and treatment. Ecancermedicalscience 7: 379.

6. Trope CG, Kaern J, Davidson B (2012) Borderline ovarian tumours. Best Pract Res Clin Obstet Gynaecol 26:325-336.

7. Kurman RJ, CM, Herrington CS, Young RH (2014) WHO classification of tumours of female reproductive organs. 4th ed. Lyon: International Agency for Research on Cancer.

8. Amortegui AJ, Sukumvanich GTP (2013) Borderline ovarian tumors - Atypical proliferative ovarian tumors. Glob Libr Women's Med.

9. Avril S, Hahn E, Specht K, Hauptmann S, Höss C, et al. (2012) Histopathologic features of ovarian borderline tumors are not predictive of clinical outcome. Gynecol Oncol 127: 516-524.

10. Uzan C, Muller E, Kane A, Rey A, Gouy S, et al. (2014) Prognostic factors for recurrence after conservative treatment in a series of 119 patients with stage I serous borderline tumors of the ovary. Ann Oncol 25: 166-171.

11. Engelen MJ, de Bruijn HW, Hollema $\mathrm{H}$, ten Hoor KA, Willemse $\mathrm{PH}$, et al. (2000) Serum CA 125, carcinoembryonic antigen and CA 19-9 as tumor markers in borderline ovarian tumors. Gynecol Oncol 78: 16-20.
12. Micci F, Haugom L, Ahlquist T, Andersen HK, Abeler VM, et al. (2010) Genomic aberrations in borderline ovarian tumors. J Transl Med 8: 21.

13. Sanz Casla MT (2003) Role of microsatellite instability in borderline ovarian tumors. Anticancer Res 23: 5139-5141.

14. Shih le M, Kurman R.J (2005) Molecular pathogenesis of ovarian borderline tumors: New insights and old challenges. Clin Cancer Res 11: 7273-7279.

15. Ho CL, Kurman RJ, Dehari R, Wang TL, Shih leM (2004) Mutations of BRAF and KRAS precede the development of ovarian serous borderline tumors. Cancer Res 64: 6915-6918.

16. Anglesio MS, Arnold JM, George J, Tinker AV, Tothill R, et al. (2008) Mutation of ERBB2 provides a novel alternative mechanism for the ubiquitous activation of RAS-MAPK in ovarian serous low malignant potential tumors. Mol Cancer Res 6: 1678-1690.

17. Hannibal CG, Vang R, Junge J, Frederiksen K, Kjaerbye-Thygesen A, et al (2014) A nationwide study of serous "borderline" ovarian tumors in Denmark 1978-2002: Centralized pathology review and overall survival compared with the general population. Gynecol Oncol 134: 267-273.

18. Giurgea LN, Ungureanu C, Mihailovici MS (2012) The immunohistochemical expression of p53 and Ki67 in ovarian epithelial borderline tumors. Correlation with clinicopathological factors. Rom J Morphol Embryol 53: 967-973.

19. Berchuck A, Kohler MF, Marks JR, Wiseman R, Boyd J, et al. (1994) The p53 tumor suppressor gene frequently is altered in gynecologic cancers. Am J Obstet Gynecol 170: 246-252.

20. Singer $G$ (2005) Patterns of p53 mutations separate ovarian serous borderline tumors and low- and high-grade carcinomas and provide support for a new mode of ovarian carcinogenesis: A mutational analysis with immunohistochemical correlation. Am J Surg Pathol 29: 218-224.

21. Whittemore AS, McGuire V (1997) Ovulation, p53 mutations, and ovarian cancer--a causal link? J Natl Cancer Inst 89:906-907.

22. Grisham RN, lyer G, Sala E, Zhou Q, lasonos A, et al. (2014) Bevacizumab shows activity in patients with low-grade serous ovarian and primary peritoneal cancer. Int J Gynecol Cancer 24:1010-1014.

23. Morice P, Uzan C, Fauvet R, Gouy S, Duvillard P, et al. (2012) Borderline ovarian tumour: pathological diagnostic dilemma and risk factors for invasive or lethal recurrence. Lancet Oncol 13: e103-115. 